\title{
Staves and Petals: Multi-module Local Support Structures of the ATLAS ITk Strips Upgrade
}

\author{
Daniel Rodríguez Rodríguez ${ }^{1}$ and Carlos García Argos ${ }^{2}$, on behalf of the \\ ATLAS ITk Collaboration \\ 1 Instituto de Física Corpuscular (Valencia, Spain) Daniel.Rodriguez@ific.uv.es \\ ${ }^{2}$ University of Freiburg (Germany) carlos.garcia.argos@cern.ch
}

\begin{abstract}
The ATLAS Inner Tracker (ITk) is an all-silicon tracker that will replace the existing inner detector at the Phase-II Upgrade of ATLAS. The outermost part of the tracker consists of the strip tracker, in which the sensor elements consist of silicon micro-strip sensors with strip lengths varying from 1.7 to up to $10 \mathrm{~cm}$. The current design is part of the ATLAS ITk Strip Detector Technical Design Report (TDR) and envisions a four-layer barrel and two six-disk end-cap regions. The sensor and readout units (modules) are directly glued onto multi-module, lowmass, high thermal performance carbon fibre structures, called staves for the barrel and petals for the end-cap. They provide cooling, power, data and control lines to the modules with a minimal amount of external services. An extensive prototyping program was put in place over the last years to fully characterise these structures mechanically, thermally, and electrically. Thermo-mechanical stave and petal prototypes have recently been built and are currently under intensive study. This contribution will focus on describing the stave and petal structures and the prototyping work carried out so far. In addition, some details of the work carried out on the global supports which will hold the staves and petals in place will also be presented.
\end{abstract}

Keywords: ATLAS ITk, strips detector, mechanics

\section{Introduction}

The ATLAS ITk strips detector will be built using multi-module structures called staves and petals, which act as local supports. These structures are then assembled in the larger structures, or global supports, called cylinders and endcap discs. This contribution is focused on the status of the local supports design as given in the Technical Design Report [1].

\section{Local Supports}

The local supports provide mechanical stability and services to the modules. Services are cooling, power, sensor bias and data transmission. The support 
consists of a core with electric polyimide bus-tapes co-cured together with carbon fibre skins at the core surface. The core is made of carbon fibre honeycomb with titanium cooling pipes. A cross section of a stave, which is the final assembled object combining the detector modules and the core, is shown in Fig. 1.

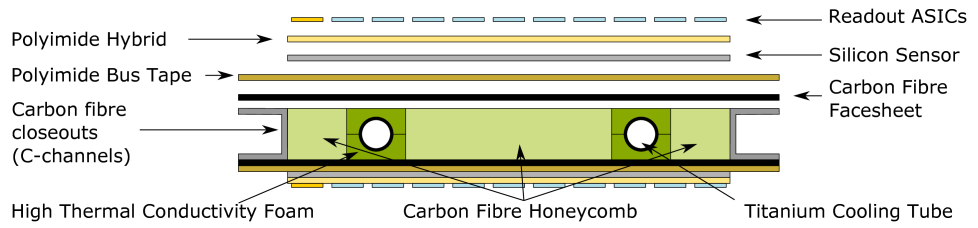

Fig. 1. Cross section of a local support structure.

The modules are glued on the bus-tape and the electrical connections are done via wire-bonds to the bus-tape. The connections to the services outside of the tracker are realised via optical fibre for data, copper wires for power and titanium pipes for cooling.

\subsection{Mechanical and Thermal Performance}

There are several general performance requirements for the local supports. Primarily, they must ensure geometric stability, which will determine the tracking performance. In addition, the thermal properties must be such that the detector is capable of operating at the required temperature during its lifetime.

The staves and petals will be cooled using $\mathrm{CO}_{2}$, which will drive the temperature down to $-35^{\circ} \mathrm{C}$ during normal operation and $-55^{\circ} \mathrm{C}$ under fault conditions. With warm temperatures of around $20^{\circ} \mathrm{C}$ this means the supports will operate with thermal excursions of up to $75 \mathrm{~K}$.

In order to avoid thermal runaway of the sensors, the thermal performance of the supports must ensure a good thermal path from the sensors to the coolant in the pipes.

Thermo-mechanical prototypes have been built and measured in order to establish the suitability of the designs. An example of these measurements is shown in Fig. 2, which depicts the infra-red image of the thermo-mechanical petal while both sides were powered and cooled down to $-20^{\circ} \mathrm{C}$ [1]. The hot spots correspond to the DC-DC converters and the Hybrid Controller Chips. These results are in good qualitative agreement with the simulations.

\subsection{Electrical Performance}

The local supports have to be capable of carrying the required power to the modules. For the low voltage, a maximum voltage drop of $1 \mathrm{~V}$ is allowed on both 


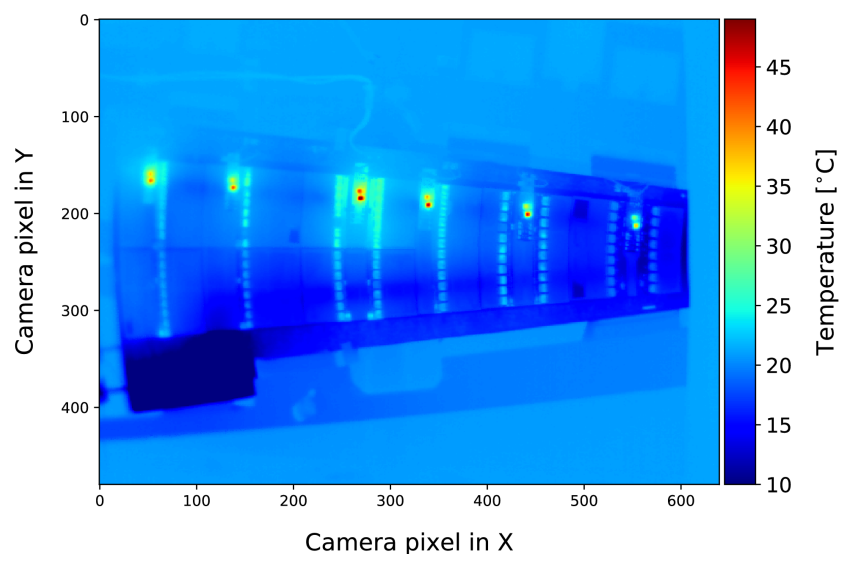

Fig. 2. Thermal image of the thermo-mechanical petal.

staves and petals, with maximum currents of $8.2 \mathrm{~A}$. This takes into account the current increase during the TID peak [2].

For sensor bias voltage, the maximum voltage has to be $750 \mathrm{~V}$ with current as high as $100 \mathrm{~mA}$.

In addition, there are three different types of signals transmitted over the bus-tape [3]:

- Timing, Trigger and Control (TTC) signals: a $40 \mathrm{MHz}$ clock and two data lines at $160 \mathrm{MHz}$. They are multi-drop, differential lines, split into sections that have between two and ten capacitive loads. The measurement for the worst case is shown in Fig. 3, which corresponds to a Bit Error Ratio (BER) of less than $10^{-12}$.

- Data from the detector modules: point-to-point $640 \mathrm{Mbps}$ links with 6b8b or $8 \mathrm{~b} 10 \mathrm{~b}$ encoding on differential lines. The staves require 28 links whereas the petals require 14 links on each bus-tape. Figure 4 shows the eye diagram for a $620 \mathrm{Mbps}$ signal. The measurements were also performed at $777 \mathrm{Mbps}$, with a resulting BER of less than $10^{-13}$.

- Monitoring and slow control data are sent using I2C at a maximum rate of $400 \mathrm{kHz}$, using single-ended pairs.

\subsection{Amount of Material}

An estimate of the material for a short-strip barrel stave and for a petal are shown in Tab. 1. The numbers are calculated based upon current stave and petal prototyping and extrapolated to the use of expected lower-mass components. The total numbers of the radiation length are $1.8 \%$ in the barrel layers, compared to $2.48 \%$ in the current SCT, and $1.78 \%$ for the end-cap discs, compared to $3.28 \%$ in the current SCT end-cap region [4]. 


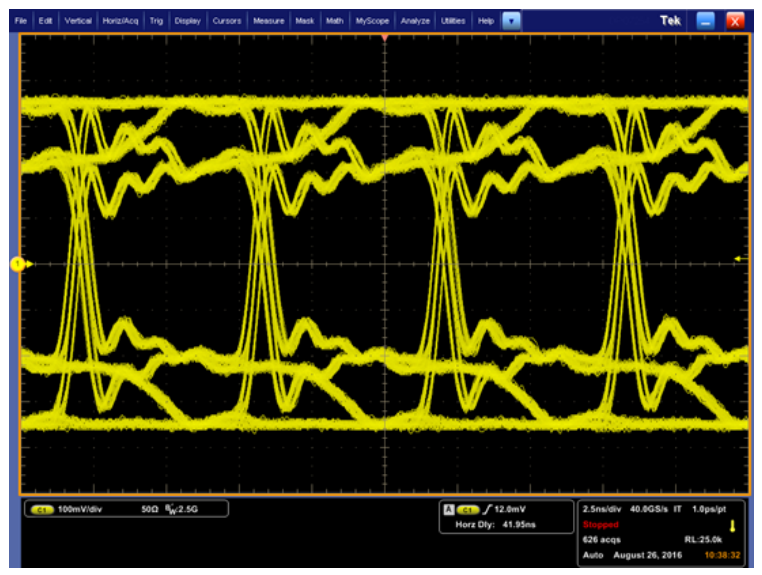

Fig. 3. Eye diagram of a Pseudo-Random Binary Sequence pattern at $160 \mathrm{Mbps}$ on a line with 10 loads.

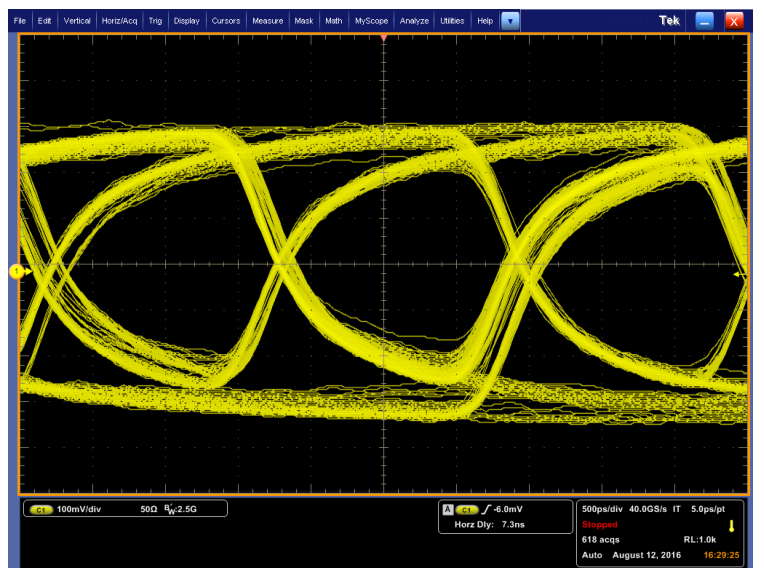

Fig. 4. Eye diagram of a 8b10b pattern at $620 \mathrm{Mbps}$ on a $1.4 \mathrm{~m}$ long bus-tape.

A reduction in the amount of material can be achieved as a result of the high degree of sharing of support, power and services in the designs for the ITk.

\section{Conclusions}

The engineering designs of the stave and petal cores are well advanced, with adequate mechanical and thermal performance of the cores for the ITk. In addition, the electrical performance of the first bus-tape designs exceed the requirements. 
Table 1. Material amount estimations for barrel staves and end-cap petals.

\begin{tabular}{lc|lc}
\multicolumn{2}{c}{ Barrel } & \multicolumn{2}{c}{ End-Cap } \\
\hline Component & Radiation Length [\%] & Component & Radiation Length [\%] \\
\hline Stave Core & 0.48 & Petal Core & 0.46 \\
Bus Cable & 0.18 & Bus cables & 0.23 \\
Short-Strip Modules & 1.08 & Modules & 1.04 \\
Module Adhesive & 0.06 & Module adhesive & 0.05 \\
\hline Total & 1.80 & Total & 1.78 \\
\hline
\end{tabular}

\section{References}

1. ATLAS Collaboration: Technical Design Report for the ATLAS Inner Tracker Strip Detector. CERN-LHCC-2017-005, April 2017.

2. A. La Rosa: Irradiation induced effects in the FE-I4 front-end chip of the ATLAS IBL detector. IEEE NSS/MIC, Strasbourg, France, 2016.

3. J. Dopke et al.: Lessons learned in high frequency data transmission design: ATLAS strips bus tape, JINST 12 (2017) no.01, C01019. doi:10.1088/17480221/12/01/C01019

4. ATLAS Collaboration: The ATLAS Experiment at the CERN Large Hadron Collider, JINST 3 (2008) S08003. 